\title{
Faster and More Focused Control-Flow Analysis for Business Process Models Through SESE Decomposition
}

\author{
Jussi Vanhatalo ${ }^{1,2}$, Hagen Völzer ${ }^{1}$, and Frank Leymann ${ }^{2}$ \\ 1 IBM Zurich Research Laboratory, Säumerstrasse 4, CH-8803 Rüschlikon, Switzerland \\ \{juv,hvo\}@zurich.ibm.com \\ 2 Institute of Architecture of Application Systems, University of Stuttgart \\ Universitätsstrasse 38, D-70569 Stuttgart, Germany \\ frank. leymann@iaas.uni-stuttgart.de
}

\begin{abstract}
We present a technique to enhance control-flow analysis of business process models. The technique considerably speeds up the analysis and improves the diagnostic information that is given to the user to fix control-flow errors. The technique consists of two parts: Firstly, the process model is decomposed into single-entry-single-exit (SESE) fragments, which are usually substantially smaller than the original process. This decomposition is done in linear time. Secondly, each fragment is analyzed in isolation using a fast heuristic that can analyze many of the fragments occurring in practice. Any remaining fragments that are not covered by the heuristic can then be analyzed using any known complete analysis technique.

We used our technique in a case study with more than 340 real business processes modeled with the IBM WebSphere Business Modeler. The results suggest that control-flow analysis of many real process models is feasible without significant delay (less than a second). Therefore, control-flow analysis could be used frequently during editing time, which allows errors to be caught at earliest possible time.
\end{abstract}

\section{Introduction}

The quality of a business process model becomes crucial when it is executed directly on a workflow engine or when it is used for generating code that is to be executed. A correct model is also important when one tries to obtain realistic business measures from a process model through simulation. Detecting and fixing errors as early as possible can therefore substantially reduce costs.

The control flow of a business process can be modeled as a workflow graph [1114]. A workflow graph that has no structural errors such as deadlocks or lack of synchronization [11] is said to be sound [14]. Soundness can and should be checked automatically during the modeling phase. To achieve a high acceptance among the users, the soundness check should

- be as fast as possible and not delay the process of constructing the model - note that a fast soundness check that can be done after each small change of the model allows the user to identify the change that introduced an error — and

- produce useful diagnostic information that helps to locate and fix errors. 
When reviewing the techniques currently available for deciding soundness, there seems to be a trade-off between the two requirements. The fastest technique known (cf. [146]) translates the workflow graph into a free choice Petri net (cf. [2]) and then decides soundness of that Petri net using the rank theorem (cf. [2]). This technique uses time that is cubic in the size of the workflow graph, but does not provide useful diagnostic information. The best diagnostic information is currently provided by a search of the state space of the workflow graph. This can return an execution sequence that leads to the error but it can use time that is exponential in the size of the workflow graph. Esparza [3] (cf. also [2]) provides a technique that can be used to decide soundness in polynomial time (more than cubic) which could potentially provide some diagnostic information, but the latter has not yet been worked out. The analysis tool Woflan [17] can decide soundness and provide diagnostic information, but because the tool ultimately resorts to state space search, it can also take exponential time.

Some authors provide algorithms for deciding soundness for the special case of acyclic workflow graphs. Perumal and Mahanti [10] gave an algorithm that takes quadratic time, which improves on previous approaches for that special case, which were either slower [7] or incomplete [11].

Given any complete technique for deciding soundness from above, we propose two enhancements in this paper. Firstly, we propose to decompose the workflow graph into a tree of single-entry-single-exit (SESE) fragments. This technique is known from compiler theory and can be done in linear time [5]. To check soundness of the workflow graph, one can now check soundness of each fragment in isolation. The overall time used now depends mainly on the size of the largest fragment. We show by experimental evidence on a large number of industrial workflow models that the largest fragment of a workflow graph is usually considerably smaller than the workflow graph itself.

Zerguini [18] and Hauser et al. [4] have proposed similar techniques of deciding soundness through decomposition into fragments. However, they decompose into multiple-entry-multiple-exit (MEME) fragments. These fragments are more general, and include SESE fragments as a special case. This however implies that a fragment can be less intuitive in general. Moreover, their decomposition into fragments is no longer unique and their decomposition algorithms are slower; while Zerguini's algorithm [18] uses quadratic time, the time complexity of the approach of Hauser et al. [4] is unknown, but we conjecture it to be at least quadratic. Both techniques could be used after our fast SESE decomposition.

A nice feature of the decomposition (SESE or MEME) approach is that each error is contained in a fragment. Thus, the error can be shown in a small local context, which in turn should help fixing the error. Errors that are located in disjoint fragments are likely to be independent. Hence, the decomposition also allows multiple independent errors to be detected in one pass.

The second enhancement we propose are two heuristics that can prove soundness or unsoundness of some fragments in linear time. The heuristics are meant to be used before any of the complete techniques from the literature are used, because the latter are likely to be more expensive. The heuristics are based on the observation that many of the fragments found in real process models have a simple structure that can be recognized quickly. The first heuristic uses ideas from Hauser et al. [4]. 
Note that simple reduction rules (e.g. [2|11[15]) can also be used to speed up the verification. Usually applied with low cost, they reduce the process model while preserving soundness.

We have implemented our technique and tried it on two libraries of altogether more than 340 industrial process models. $81 \%$ of the process models can be completely analyzed with the SESE decomposition and the heuristics alone. For the remaining cases, the analysis task becomes considerably smaller through SESE decomposition.

Mendling et al. [98] have analyzed more than 2000 EPC process models using the Woflan tool [17] for a relaxed version of soundness. We are not aware of any other published case study with large industrial data.

This paper is structured as follows. In Sect. 2] we recall the definition of workflow graphs and their soundness. Section 3 describes our approach in detail. Section 4 presents the results of the case study. Missing proofs can be found in a technical report [16].

\section{Sound Workflow Graphs}

In this section, we recall the definition of sound workflow graphs [1114]. We also give an equivalent characterization of soundness, which will be used later in this paper.

\subsection{Workflow Graphs}

A workflow graph is a directed graph $G=(N, E)$, where a node $n \in N$ is exactly one of the following: a start node, a stop node, an activity, a fork, a join, a decision, or a merge such that

1. there is exactly one start node and exactly one stop node; the start node has no incoming edges and exactly one outgoing edge, whereas the stop node has exactly one incoming edge but no outgoing edges;

2. each fork and each decision has exactly one incoming edge and two or more outgoing edges, whereas each join and each merge has exactly one outgoing edge and two or more incoming edges; each activity has exactly one incoming and exactly one outgoing edge;

3. each node $n \in N$ is on a path from the start node to the stop node.

It follows from the definition that no node is directly connected to itself. Figure 1 shows an example of a workflow graph. An activity is depicted as a square, a fork and a join as a thin rectangle, a decision as a diamond, and a merge as a triangle. Start and stop nodes are depicted as (decorated) circles. The unique outgoing edge of the start node is called the entry edge, and the unique incoming edge of the stop node is

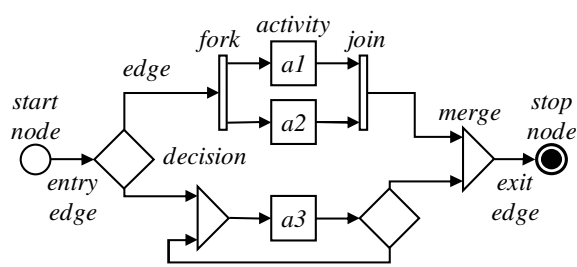

Fig. 1. A workflow graph called the exit edge of the workflow graph. 
The semantics of a workflow graph is, similarly to Petri nets, defined as a token game. A state of a workflow graph is represented by tokens on the edges of the graph. Let $G=(N, E)$ be a workflow graph. A state of $G$ is a mapping $s: E \rightarrow \mathbb{N}$, which assigns a natural number to each edge. When $s(e)=k$, we say that edge $e$ carries $k$ tokens in state $s$. The semantics of the various nodes is defined as usual. An activity, a fork, and a join remove one token from each of its ingoing edges and add one token to each of its outgoing edges. A decision node removes a token from its incoming edge, nondeterministically chooses one of its outgoing edges, and adds one token to that outgoing edge. A merge node nondeterministically chooses one of its incoming edges on which there is at least one token, removes one token from that edge, and adds a token to its outgoing edge.

To be more precise, let $s$ and $s^{\prime}$ be two states and $n$ a node that is neither a start nor a stop node. We write $s \stackrel{n}{\rightarrow} s^{\prime}$ when $s$ changes to $s^{\prime}$ by executing $n$. We have $s \stackrel{n}{\rightarrow} s^{\prime}$ if

1. $n$ is an activity, fork or join and

$$
s^{\prime}(e)= \begin{cases}s(e)-1 & e \text { is an incoming edge of } n, \\ s(e)+1 & e \text { is an outgoing edge of } n, \\ s(e) & \text { otherwise. }\end{cases}
$$

2. $n$ is a decision and there exists an outgoing edge $e^{\prime}$ of $n$ such that

$$
s^{\prime}(e)= \begin{cases}s(e)-1 & e \text { is an incoming edge of } n, \\ s(e)+1 & e=e^{\prime} \\ s(e) & \text { otherwise. }\end{cases}
$$

3. $n$ is a merge and there exists an incoming edge $e^{\prime}$ of $n$ such that

$$
s^{\prime}(e)= \begin{cases}s(e)-1 & e=e^{\prime}, \\ s(e)+1 & e \text { is an outgoing edge of } n, \\ s(e) & \text { otherwise. }\end{cases}
$$

Node $n$ is said to be activated in a state $s$ if there exists a state $s^{\prime}$ such that $s \stackrel{n}{\rightarrow} s^{\prime}$. A state $s^{\prime}$ is reachable from a state $s$, denoted $s \stackrel{*}{\longrightarrow} s^{\prime}$, if there exists a (possibly empty) finite sequence $s_{0} \stackrel{n_{1}}{\rightarrow} s_{1} \ldots s_{k-1} \stackrel{n_{k}}{\rightarrow} s_{k}$ such that $s_{0}=s$ and $s_{k}=s^{\prime}$.

\subsection{Soundness}

To define soundness [14] of a workflow graph $G$, we use the following notions. The initial state of $G$ is the state that has exactly one token on the entry edge and no tokens elsewhere. The terminal state of $G$ is the state that has exactly one token on the exit edge and no tokens elsewhere. A stopping state of $G$ is a state of $G$ in which the exit edge carries at least one token.

$G$ is live if for every state $s$ that is reachable from the initial state, a stopping state is reachable from s. $G$ is safe if the terminal state is the only stopping state that is reachable from the initial state. $G$ is sound if it is live and safe. The soundness criterion is a global view on correctness. Liveness says that each run can be completed, and safeness says that each completion of a run is a proper termination, i.e., there are no tokens inside the graph upon completion. The workflow graph in Fig. 11 is sound. Figure 2 shows simple examples of unsound graphs. The graph in part (a) is not live, the graph in part (b) is not safe. 


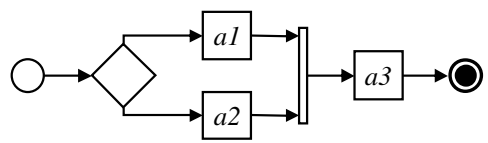

(a)

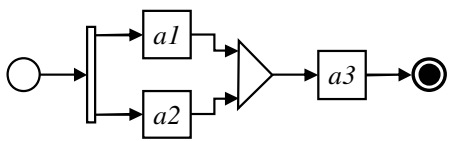

(b)

Fig. 2. Structural conflicts: (a) a local deadlock (b) a lack of synchronization

The two examples of unsound workflow graphs in Fig. 2 are examples of a structural conflict, viz. a local deadlock (part a) and a lack of synchronization (part b) [11]. A local deadlock is a state $s$ such that there exists a join $n$ where (i) at least one incoming edge of $n$ carries a token in $s$ and (ii) there is an incoming edge $e$ of $n$ such that $e$ does not carry a token in any state $s^{\prime}$ that is reachable from $s$. That is, that join will never get 'enough' tokens. A state $s$ of $G$ has lack of synchronization if there is a merge $n$ such that more than one incoming edge of $n$ carries a token, i.e., that merge gets 'too many' tokens. Note that a lack of synchronization can lead to a state where there is more than one token on a single edge. Van der Aalst et al. [14] have shown that for acyclic workflow graphs, soundness is equivalent with the condition that neither a local deadlock nor a state with lack of synchronization is reachable from the initial state. We generalize this here for arbitrary workflow graphs, therefore providing a local view of correctness for arbitrary workflow graphs.

Definition 1. Let $G$ be a workflow graph. $G$ is locally live if there is no local deadlock that is reachable from the initial state. $G$ is locally safe if no state is reachable from the initial state that has more than one token on a single edge.

Theorem 1. A workflow graph is sound if and only if it is locally safe and locally live.

\section{Enhanced Control-Flow Analysis}

In this section, we explain the decomposition of a workflow graph into SESE fragments and show how some fragments can be quickly recognized as sound or unsound.

\subsection{Decomposition into Fragments}

Figure 3 shows a workflow graph and its decomposition into SESE fragments (cf. e.g. [5]). A SESE fragment is depicted as a dotted box. Let $G=(N, E)$ be a workflow graph. A SESE fragment (fragment for short) $F=\left(N^{\prime}, E^{\prime}\right)$ is a nonempty subgraph of $G$, i.e., $N^{\prime} \subseteq N$ and $E^{\prime}=E \cap\left(N^{\prime} \times N^{\prime}\right)$ such that there exist edges $e, e^{\prime} \in E$ with $E \cap\left(\left(N \backslash N^{\prime}\right) \times N^{\prime}\right)=\{e\}$ and $E \cap\left(N^{\prime} \times\left(N \backslash N^{\prime}\right)\right)=\left\{e^{\prime}\right\} ; e$ and $e^{\prime}$ are called the entry and the exit edge of $F$, respectively.

The workflow graph shown in Fig. 3 has more fragments than those that are shown explicitly. For example, the union of fragments $J$ and $K$, denoted $J \cup K$, as well as $K \cup L$ are fragments. Those however are not of interest here and they are subsumed in fragment $X$. Interesting fragments will be called canonical, which are defined in the following. We say that two fragments $F$ and $F^{\prime}$ are in sequence if the exit edge of $F$ is 


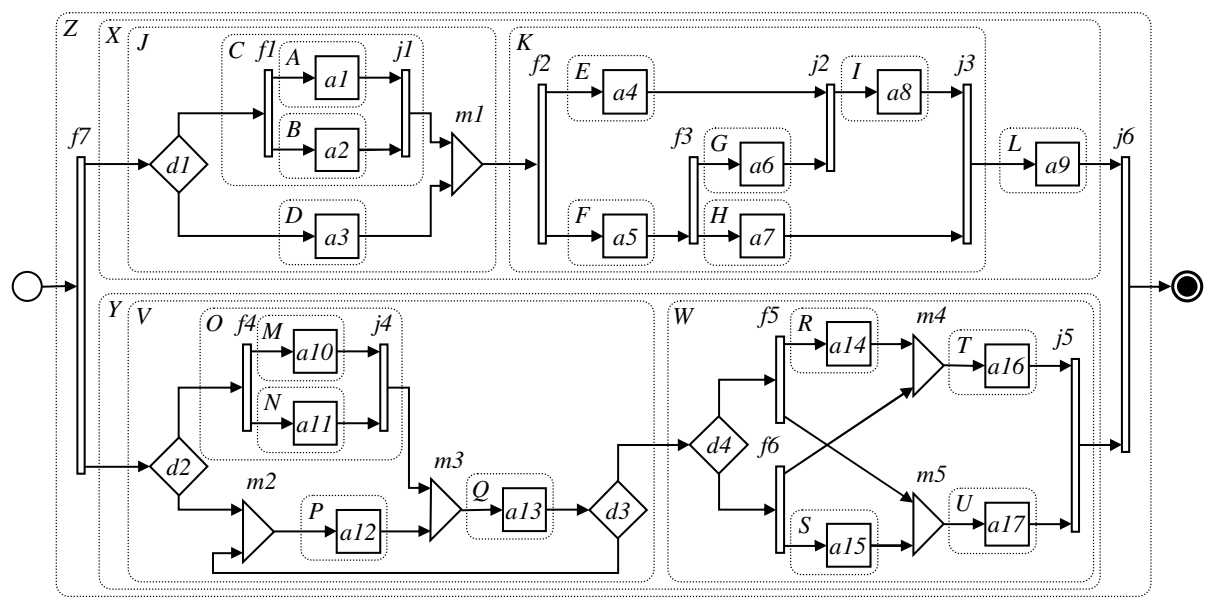

Fig. 3. Decomposition of a workflow graph into canonical fragments

the entry edge of $F^{\prime}$ or vice versa. The union $F \cup F^{\prime}$ of two fragments $F$ and $F^{\prime}$ that are in sequence is a fragment again. A fragment $F$ is non-canonical if there are fragments $X, Y, Z$ such that $X$ and $Y$ are in sequence, $F=X \cup Y$, and $F$ and $Z$ are in sequence; otherwise $F$ is said to be canonical.

The fragments shown in Fig. 3 are exactly the canonical fragments of that workflow graph. Canonical fragments do not overlap. Two canonical fragments are either nested or disjoint [5]. Therefore, it is possible to organize the canonical fragments in a unique tree, similarly to the Program Structure Tree shown in [5]. We call this tree the process structure tree of a workflow graph. It can be computed in time linear in the size of the workflow graph [5 1]. As we are only interested in canonical fragments, we mean 'canonical fragment' whenever we say 'fragment' in the following.

\section{Z}

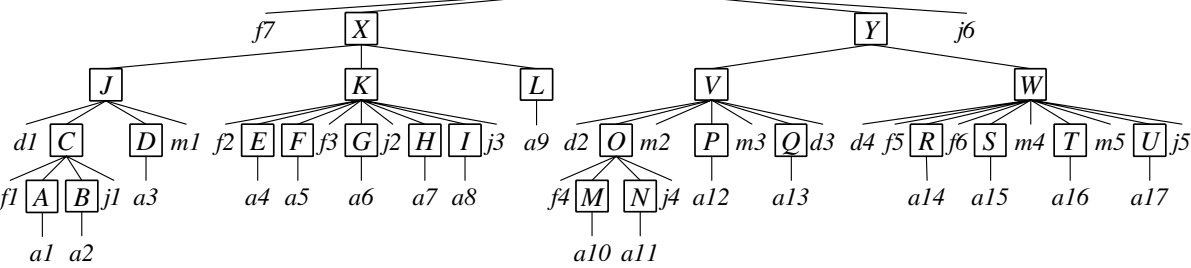

Fig. 4. The process structure tree of the workflow graph in Fig. 3

Figure 4 shows the process structure tree of the workflow graph from Fig. 3. A fragment is represented as a boxed tree node. In addition, we represent the nodes of the workflow graph as leaves in the tree. The parent of a fragment $F$ (a workflow graph

\footnotetext{
${ }^{1}$ Note: Ananian [1] gives a slightly modified linear time algorithm that includes corrections.
} 
node $n$ ) is the smallest fragment $F^{\prime}$ that contains $F(n)$. Then, we also say that $F$ is a child fragment of $F^{\prime}$ ( $n$ is a child node of $\mathrm{F}^{\prime}$ ).

To check the soundness of a workflow graph, it is sufficient to analyze the soundness of its fragments in isolation. Note that a fragment can be viewed as a workflow graph by adding entry and exit edges as well as a start and a stop node. Hence we can apply the notion of soundness also to fragments. The following theorem follows from classical Petri net theory (e.g. [12], cf. also [13[14[18]).

Theorem 2. A workflow graph is sound if and only if all its child fragments are sound and the workflow graph that is obtained by replacing each child fragment with an activity is sound.

Checking soundness of fragments can therefore be done along the structure of the process structure tree, starting from the leaves upwards. If a fragment $F$ was checked for soundness, checking soundness of the parent fragment (in the tree) can abstract from the internal structure of $F$, i.e., $F$ can be treated as an activity in the parent fragment. Figure 5 shows fragments $J$ and $V$ from Figs. 3 and 4 where fragment $J$ abstracts from the structure of the child fragments $C$ and $D$ and fragment $V$ abstracts from the structure of fragment $O$.

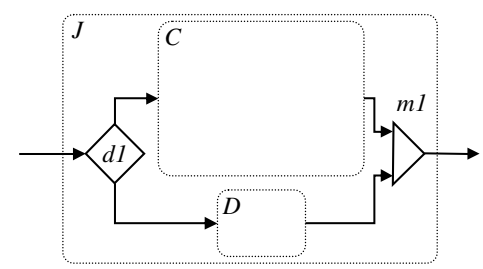

(a)

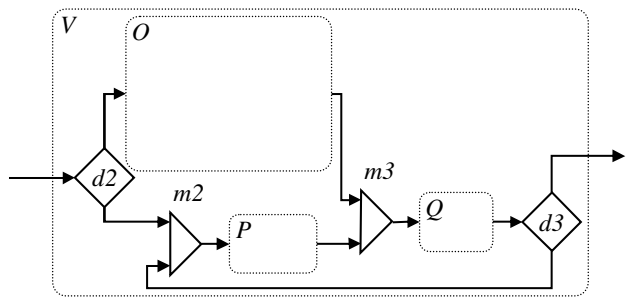

(b)

Fig. 5. Fragments $J$ and $V$ ignoring the structure of their child fragments

\subsection{Heuristic for Sound Fragments}

Many fragments that occur in practice have a simple structure that can easily be recognized, which identifies those fragments as being sound. To this end, we define the following categories, based on definitions given by Hauser et al. [4].

Definition 2. Let $F$ be a fragment of a workflow graph. $F$ is

1. well-structured if it satisfies one of the following conditions:

- F has no decisions, merges, forks or joins as children in the process structure tree (sequence),

- F has exactly one decision and exactly one merge, but no forks and no joins as children. The entry edge of $F$ is the incoming edge of the decision, and the exit edge of $F$ is the outgoing edge of the merge (sequential branching),

- F has exactly one decision and exactly one merge, but no forks and no joins as children. The entry edge of $F$ is an incoming edge of the merge, and the exit edge of $F$ is an outgoing edge of the decision (cycle), 
- F has exactly one fork, exactly one join, no decisions and no merges as children. The entry edge is the incoming edge of the fork. The exit edge is the outgoing edge of the join. (concurrent branching).

2. an unstructured concurrent fragment if $F$ is not well-structured, contains no cycles, and has no decisions and no merges as children.

3. an unstructured sequential fragment if $F$ is not well-structured and has no forks and no joins as children.

4. a complex fragment if it is none of the above.

It is easy to see that it can be decided in linear time to which of the four categories listed above a fragment belongs.

Theorem 3. If a fragment $F$ is well-structured, an unstructured concurrent, or an unstructured sequential fragment, then $F$ is sound if and only if all its child fragments are sound.

This theorem was already observed by Hauser et al. [4]. Note that all fragment categories ignore the structure of child fragments, taking only the top-level structure into account. In Fig. 3 , fragments $X$ and $Y$ are well-structured (sequence) and so are also fragments $C, O, Z$ (concurrent branching) and $J$ (sequential branching). Fragments $K$ and $V$ are examples of unstructured concurrent and unstructured sequential fragments, respectively. Note that unstructured sequential fragments may contain cycles, whereas unstructured concurrent fragments must not.

A complex fragment may be sound or unsound. Fragment $W$ in Fig. 3 is a sound complex fragment. It follows from Theorems 2 and 3 that the entire workflow graph in Fig. 3 is sound.

\subsection{Heuristic for Unsound Fragments}

Some complex fragments can be efficiently determined as not being sound:

Theorem 4. A complex fragment $F$ is not sound if it satisfies one of the following conditions:

1. F has one or more decisions (merges), but no merges (decisions) as children in the process structure tree,

2. F has one or more forks (joins), but no joins (forks) as children,

3. F contains a cycle, but has no decisions or no merges as children.

It is again easy to see that this heuristic can be applied in linear time. We actually found numerous errors in real process models using this heuristic (see Sect. 4.2). The relative strength of this heuristic is due to the fact that, similar to the heuristic in Sect. 3.2, the structure of child fragments is ignored.

\section{Case Study}

In this section, we describe the results of an application of our proposed technique in a case study with industrial data. 


\subsection{The Data}

We have analyzed the soundness of more than 340 workflow graphs that were extracted from two libraries of industrial business processes modeled in the IBM WebSphere Business Modeler. Although the modeling language used there is more expressive than workflow graphs, it was possible to translate the process models into workflow graphs because strict guidelines were used for the construction of these process models. The description of the translation is beyond the scope of this paper.

Library 1 consists of more than 140 processes. The extracted workflow graphs have, on average, 67 edges, with the maximum being 215. Library 2 is an experimental extension of Library 1 . It contains similar processes, but many features were added to the processes and also some processes were added. It contains more than 200 processes, the extracted workflow graphs have 99 edges on average, with the maximum being 342 .

\subsection{The Results}

We analyzed the libraries using an IBM ThinkPad T43p laptop that has a $2.13 \mathrm{GHz}$ Intel Pentium M processor and 2 GB of main memory. The entire Library 1 is analyzed in 9 seconds, and Library 2 in 15 seconds. Thus, the average analysis time per workflow graph is less than 0.1 seconds.

SESE Decomposition. As described in Sect. 1 the worst-case time a complete technique needs for checking the soundness of a workflow graph can be polynomial or exponential in the size $g$ of the workflow graph, which is defined to be its number of edges. Similarly, the size of a fragment is defined as its number of edges plus 2 (for the entry edge and the exit edge). If we use a complete technique after the SESE decomposition according to the procedure in Sect. 3.1 the time used is linear in the number of fragments. Note also that the number of fragments in a workflow graph is at most twice the number of nodes. The overall time used therefore mainly depends on the size $f_{\max }$ of the largest fragment to which we have to apply the complete technique. If the complete technique uses polynomial time $g^{c}$ for some constant $c$, then the reduction that SESE decomposition could achieve is $g^{c} / f_{\max }^{c}=\left(g / f_{\max }\right)^{c}$. If the complete technique uses exponential time $c^{g}$, then the possible reduction is $c^{g} / c^{f_{\max }}=c^{g-f_{\max }}$. Table 1 shows the values for $g / f_{\max }$ and $g-f_{\max }$ for Library 1 as an indication of the reduction achieved due to SESE decomposition.

Figure 6 shows the largest fragment size in relation to the graph size for each workflow graph in Library 1. It shows that the graph size has only a minor impact on the

Table 1. Graph size (i.e., number of edges) compared to the size of the largest fragment in the graph and size reductions for the workflow graphs in Library 1

\begin{tabular}{lllll}
\hline & Graph size & $\begin{array}{l}\text { Largest fragment size } \\
f_{\max }\end{array}$ & $\begin{array}{l}\text { Reduction } \\
g-f_{\max }\end{array}$ & $\begin{array}{l}\text { Reduction } \\
g / f_{\max }\end{array}$ \\
\hline Maximum & 215 & 51 & 191 & 9.0 \\
Average & 67 & 24 & 44 & 2.8 \\
Minimum & 11 & 11 & 0 & 1.0 \\
\hline
\end{tabular}




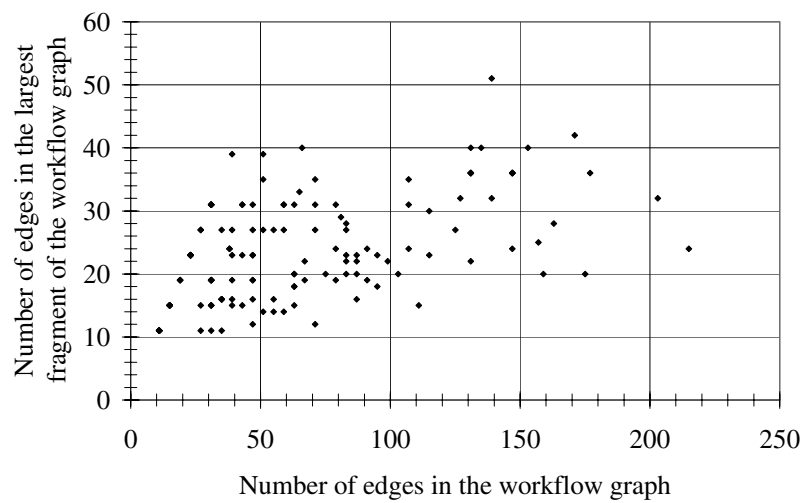

Fig. 6. Size of largest fragment in relation to graph size for all workflow graphs in Library 1

largest fragment size. Therefore, the reduction increases as the graph size increases. Thus, the technique is most useful when the complete techniques would be most time consuming. Even a small reduction can be significant, as the complete techniques for checking soundness can take a time that is cubic or exponential in the graph size.

Table 2 shows the reduction statistics for the workflow graphs in Library 2. The graphs are larger, and also the reduction is higher.

Table 2. Graph size compared to the size of the largest fragment in the graph, and size reductions for Library 2

\begin{tabular}{lllll}
\hline & Graph size & Largest fragment size \\
& $g$ & $f_{\max }$ & $\begin{array}{l}\text { Reduction } \\
g-f_{\max }\end{array}$ & $\begin{array}{l}\text { Reduction } \\
g / f_{\max }\end{array}$ \\
\hline Maximum & 342 & 82 & 328 & 24.4 \\
Average & 99 & 21 & 78 & 5.6 \\
Minimum & 12 & 6 & 5 & 1.5 \\
\hline
\end{tabular}

Using both heuristics from Sect. 3, we can decide soundness for $68.5 \%$ of the workflow graphs in Library 2. For the remaining graphs, our prototype tool highlights the complex fragments that may be unsound. A complete analysis method is needed to decide their soundness, or they can be reviewed manually. The reduction statistics for these remaining workflow graphs are shown in Table 3.

Fragment Categories. Even though our heuristics from Sect. 3 are incomplete, we were able to decide soundness for all the workflow graphs from Library 1. They are all sound.

The first column in Table 4 illustrates the distribution of fragments according to the categories defined in Sects. 3.2.3.3 for Library 1. We excluded here any fragments that are well-structured sequences from these statistics, because most fragments are sequences and those are trivially sound and thus not interesting. 
Table 3. Library 2: Graph size, largest fragment size, and reduction for the remaining $31.5 \%$ of workflow graphs for which soundness is unknown after applying our heuristics

\begin{tabular}{lllll}
\hline & Graph size & $\begin{array}{l}\text { Largest fragment size } \\
f_{\max }\end{array}$ & $\begin{array}{l}\text { Reduction } \\
g-f_{\max }\end{array}$ & $\begin{array}{l}\text { Reduction } \\
g / f_{\max }\end{array}$ \\
\hline Maximum & 334 & 82 & 284 & 10.4 \\
Average & 126 & 32 & 94 & 4.3 \\
Minimum & 40 & 12 & 25 & 1.6 \\
\hline
\end{tabular}

We can also put entire workflow graphs into the various categories. For example, a workflow graph is complex if it has at least one complex fragment. Complex graphs are further divided into those known to be not sound by applying the heuristic in Sect. 3.3 and those for which soundness is unknown. A workflow graph is unstructured if it has at least one unstructured fragment and no complex fragments. Otherwise, a graph has only well-structured fragments and it is therefore called well-structured. Column 3 of Table 4] shows the distribution of workflow graphs in Library 1 in the various categories. The last two columns present the same statistics for Library 2.

Most fragments are well-structured, which makes it attractive to analyze fragments separately. However, only a third of the workflow graphs are well-structured and there is a considerable number of sound unstructured workflow graphs. Therefore, although well-structuredness is also an appealing correctness requirement, it seems to be overly restrictive. As unstructured fragments occur often, it makes sense to detect those with fast heuristics before using a complete analysis technique. Our heuristics can decide soundness not only for many fragments, but also for a significant proportion of the workflow graphs.

In Library 2, $43.5 \%$ of the workflow graphs contain at least one complex fragment. Only one workflow graph has more than one complex fragment. $19.7 \%$ of the fragments in Library 2 are complex fragments. Our heuristic recognized $27.3 \%$ of these fragments as being unsound. We have not yet checked the soundness of the remaining complex graphs by integrating our tool with a complete analysis method. The high error rate in Library 2 is due to its experimental nature.

Table 4. Categories of fragments and workflow graphs in the libraries

\begin{tabular}{lcccc}
\hline $\begin{array}{l}\text { Fragment category / } \\
\text { Workflow graph category }\end{array}$ & $\begin{array}{l}\text { Library 1 } \\
\text { Percentage } \\
\text { of fragments }\end{array}$ & $\begin{array}{l}\text { Percentage } \\
\text { of graphs }\end{array}$ & $\begin{array}{l}\text { Library 2 } \\
\text { Percentage } \\
\text { of fragments }\end{array}$ & $\begin{array}{l}\text { Percentage } \\
\text { of graphs }\end{array}$ \\
\hline Well-structured (sound) & $54.8 \%$ & $37.5 \%$ & $65.4 \%$ & $33.3 \%$ \\
\hline Unstructured (sound) & $45.2 \%$ & $62.5 \%$ & $14.9 \%$ & $23.1 \%$ \\
- Unstructured concurrent & $1.4 \%$ & - & $6.0 \%$ & - \\
- Unstructured sequential (acyclic) & $29.2 \%$ & - & $4.4 \%$ & - \\
- Unstructured sequential (cyclic) & $14.6 \%$ & - & $4.6 \%$ & - \\
\hline Complex & $0.0 \%$ & $0.0 \%$ & $19.7 \%$ & $43.5 \%$ \\
- Complex (not sound) & $0.0 \%$ & $0.0 \%$ & $5.4 \%$ & $12.0 \%$ \\
- Complex (soundness unknown) & $0.0 \%$ & $0.0 \%$ & $14.3 \%$ & $31.5 \%$ \\
\hline
\end{tabular}




\section{Conclusion}

We proposed a technique to focus and speed up control-flow analysis of business process models that is based on decomposition into SESE fragments. The SESE decomposition could also be used for other purposes such as browsing and constructing large processes, discovery of reusable subprocesses, code generation, and others.

We also proposed a partition of the fragments into various categories, which can be computed fast. We think that tagging a fragment with its category may help to better understand the process model and may help to establish modeling patterns. It also helps to speed up the control-flow analysis as many of the correct fragments that occur in practice have a simple structure.

We plan to integrate our prototype with existing complete verification techniques and measure the impact of SESE decomposition on the analysis time. In addition, we plan to investigate the errors that occur in Library 2, together with approaches to fix them.

Acknowledgments. We thank Michael Friess for suggesting to apply SESE decomposition to workflow graphs. We thank Wil van der Aalst, Rainer Hauser, Rania Khalaf, Jana Koehler, Oliver Kopp, Jochen Küster and Ksenia Ryndina for helpful discussions and comments.

The work published in this article was partially supported by the SUPER project (http://www.ip-super.org/) under the EU 6th Framework Programme Information Society Technologies Objective (contract no. FP6-026850).

\section{References}

1. Scott Ananian, C.: The static single information form. Master's thesis, Massachusetts Institute of Technology (September 1999)

2. Desel, J., Esparza, J.: Free Choice Petri Nets. Cambridge University Press, Cambridge (1995)

3. Esparza, J.: Reduction and synthesis of live and bounded free choice Petri nets. Inf. Comput. 114(1), 50-87 (1994)

4. Hauser, R., Friess, M., Küster, J.M., Vanhatalo, J.: An incremental approach to the analysis and transformation of workflows using region trees. IEEE Transactions on Systems, Man, and Cybernetics - Part C (June 2007) (to appear, also available as IBM Research Report RZ 3693)

5. Johnson, R., Pearson, D., Pingali, K.: The program structure tree: Computing control regions in linear time. In: PLDI. Proceedings of the ACM SIGPLAN'94 Conference on Programming Language Design and Implementation, pp. 171-185. ACM Press, New York (1994)

6. Kemper, P.: Linear time algorithm to find a minimal deadlock in a strongly connected freechoice net. In: Ajmone Marsan, M. (ed.) Application and Theory of Petri Nets 1993. LNCS, vol. 691, pp. 319-338. Springer, Heidelberg (1993)

7. Lin, H., Zhao, Z., Li, H., Chen, Z.: A novel graph reduction algorithm to identify structural conflicts. In: HICSS-35 2002. Proceedings of the 35th Hawaii International Conference on System Sciences, p. 289 (2002)

8. Mendling, J.: Detection and Prediction of Errors in EPC Business Process Models. PhD thesis, Vienna University of Economics and Business Administration (WU Wien), Austria (May 2007) 
9. Mendling, J., Moser, M., Neumann, G., Verbeek, H.M.W., van Dongen, B.F., van der Aalst, W.M.P.: Faulty EPCs in the SAP reference model. In: Dustdar, S., Fiadeiro, J.L., Sheth, A. (eds.) BPM 2006. LNCS, vol. 4102, pp. 451-457. Springer, Heidelberg (2006)

10. Perumal, S., Mahanti, A.: A graph-search based algorithm for verifying workflow graphs. In: DEXA 2005. Proceedings of the 16th International Workshop on Database and Expert Systems Applications, pp. 992-996. IEEE Computer Society, Los Alamitos (2005)

11. Sadiq, W., Orlowska, M.E.: Analyzing process models using graph reduction techniques. Inf. Syst. 25(2), 117-134 (2000)

12. Valette, R.: Analysis of Petri nets by stepwise refinements. Journal of Computer and System Sciences 18(1), 35-46 (1979)

13. van der Aalst, W.M.P.: Workflow verification: Finding control-flow errors using Petri-netbased techniques. In: van der Aalst, W.M.P., Desel, J., Oberweis, A. (eds.) Business Process Management. LNCS, vol. 1806, pp. 161-183. Springer, Heidelberg (2000)

14. van der Aalst, W.M.P., Hirnschall, A. (Eric) Verbeek, H.M.W.: An alternative way to analyze workflow graphs. In: Pidduck, A.B., Mylopoulos, J., Woo, C.C., Ozsu, M.T. (eds.) CAiSE 2002. LNCS, vol. 2348, pp. 535-552. Springer, Heidelberg (2002)

15. van Dongen, B.F., van der Aalst, W.M.P., Verbeek, H.M.W.: Verification of EPCs: Using reduction rules and Petri nets. In: Pastor, Ó., Falcão e Cunha, J. (eds.) CAiSE 2005. LNCS, vol. 3520, pp. 372-386. Springer, Heidelberg (2005)

16. Vanhatalo, J., Völzer, H., Leymann, F.: Faster and more focused control-flow analysis for business process models though SESE decomposition. IBM Research Report RZ 3694 (July 2007)

17. (Eric) Verbeek, H.M.W., Basten, T., van der Aalst, W.M.P.: Diagnosing workflow processes using Woflan. Comput. J. 44(4), 246-279 (2001)

18. Zerguini, L.: A novel hierarchical method for decomposition and design of workflow models. Journal of Integrated Design and Process Science 8(2), 65-74 (2004) 